\title{
Protective effect of vitamin E on ischaemia-reperfusion injury in ovarian grafts
}

\author{
D. Nugent ${ }^{1}$, H. Newton ${ }^{1}$, L. Gallivan ${ }^{2}$ and R. G. Gosden ${ }^{1}$ \\ ${ }^{1}$ Centre for Reproduction, Growth and Development, University of Leeds, Leeds LS2 9NS, UK; and \\ ${ }^{2}$ Department of Clinical Medicine, Clinical Sciences Building, St James's University Hospital, Leeds LS9 7TF, UK
}

\begin{abstract}
Ovarian cortical tissue cryopreservation with subsequent autografting is a potential strategy for the preservation of fertility in patients undergoing systemic chemotherapy and pelvic radiotherapy. Non-vascular implants are first subjected to a period of ischaemia before revascularization and are, therefore, vulnerable to ischaemiareperfusion injury from reactive oxygen species. Ischaemia-reperfusion injury was investigated during the first week after surgery in murine ovarian grafts and human ovarian xenografts in mice with severe combined immune deficiency (SCID) by measuring total lipid peroxides and malondialdehyde concentrations with a colorometric assay. The effects of administering an antioxidant, vitamin $\mathrm{E}$, on these concentrations were also tested. Products of lipid peroxidation were higher in nonsupplemented murine autografts compared with control ovaries $(P<0.05)$, and were significantly reduced on day 3 by vitamin $\mathrm{E}$ administration $(P<0.05)$. Similarly, in human xenografts, there was a significant reduction in lipid peroxidation with vitamin $\mathrm{E}$ administration. These results correspond to a significantly greater total follicle survival in the murine grafts of the supplemented group ( 45 versus $72 \% ; P<0.05$ ). They suggest that antioxidant treatment improves the survival of follicles in ovarian grafts by reducing ischaemia-reperfusion injury.
\end{abstract}

\section{Introduction}

As long-term survival rates for many malignant diseases steadily improve, the late effects of chemotherapy on the ovaries assume greater significance and are stimulating fresh efforts to preserve fertility (Apperley and Reddy, 1995). Sometimes there is only a temporary lull in menstrual rhythmicity, but when treatment is particularly aggressive or the patient is $>30$ years of age, the possibility of permanent sterility is high. Oophoropexy is sometimes helpful in avoiding exposure to the radiation field (Hunter et al., 1980), but it offers no protection against chemotherapy and the ovaries may absorb scattered rays. Furthermore, even if this manoeuvre is successful in minimizing radiation injury, assisted conception will probably still be required.

In theory, the only other options available for these patients are collection and storage of oocytes, embryos or ovarian tissue, or third-party egg donation. Ovarian tissue freezing has several potential advantages over oocyte and embryo freezing. Mature oocytes can only be collected at a specific time in the menstrual cycle or, more usually, after gonadotrophin administration, and they are never available in large numbers. In addition, there have been few reports of successful cryopreservation, thawing and fertilization of mature human oocytes (Parks and Ruffing, 1992; Trounson

Received 26 January 1998. and Bongso, 1996). In contrast, the cryopreservation of embryos is relatively efficient (Trounson and Bongso, 1996), but is only an option for patients from whom mature eggs can be collected and fertilized. Neither of these problems is presented by storing immature oocytes. If ovarian tissue could be cryopreserved before patients receive cancer treatment and then returned successfully after remission, there is the potential for the natural state to be restored and even for conception to be achieved in vivo with their own germ cells.

Since the initial cryopreservation techniques pioneered in spermatozoa during the 1950s, more efficient cryoprotectants and the lower temperature of liquid nitrogen have now contributed to the successful storage of murine ovarian tissue or isolated primordial follicles (Carroll and Gosden, 1993; Harp et al., 1994). In both, the follicles resumed growth and fertility returned after isografting to sterilized hosts. Recently, this technique has been extended to farm animals, whose ovaries resemble more closely those of humans (Gosden et al., 1994). Furthermore, the survival of human ovarian follicles has been demonstrated after freeze-thawing in various cryoprotectants and subsequent grafting into SCID mice (Newton et al., 1996). However, in a quantitative comparison of fresh tissue with unfrozen grafts, about $25-30 \%$ of the primordial follicles were lost as a result of grafting per se. In isologous murine grafts, approximately $50 \%$ of the follicle population remained, although the percentage varied between strains (Jones and 
Krohn, 1960; Felicio et al., 1983). A recent study reported follicle populations as high as $63 \%$ in fresh grafts of young B6CB/F1 hybrid mice (Candy et al., 1997).

These follicular losses can be attributed, at least partly, to ischaemia-reperfusion injury resulting from lipid peroxidation and other free radical mediated effects in the grafts. Lipid peroxidation involves damage to the cell membrane as a result of formation of lipid peroxides and their by-products, such as aldehydes. Malondialdehyde is an end-product derived from the breakdown of polyunsaturated fatty acids and related esters and is often used as an indicator of lipid peroxidation (Esterbauer and Cheeseman, 1990). It is well established that lipid peroxidation arises with solid organ transplantation during ischaemia-reperfusion injury (Gutteridge and Halliwell, 1994) and media containing allopurinol and antioxidants are used to quench free radicals and preserve organs before transplantation (Lindell et al., 1991). Ovarian grafting has not been practised clinically for many years (Nugent et al., 1997), but current interest in reviving the technique for use with cryopreserved tissue has prompted investigation of whether lipid peroxidation is a problem in ovarian grafts and, if so, whether it can be reduced. Vitamin $E$ is an antioxidant that breaks the lipoperoxidative chain reaction by interfering with lipid peroxyl and alkoxyl radicals (Krinsky, 1988). In this study, lipid peroxides in ovarian grafts were measured, follicle loss was quantified and the potential benefits of administering vitamin $\mathrm{E}$ ( $\alpha$-tocopherol) were tested.

\section{Materials and Methods}

\section{Experimental surgery}

Balb/c mice were obtained from the laboratory breeding stock in Biomedical Services at the University of Leeds and were maintained on a standard pelleted diet and water. All experimental surgery was conducted with halothane anaesthesia under a project licence issued by the UK Home Office. In animals undergoing ovarian grafting, the ovary was ligated at its pedicle and was removed intact. After bisection, the ovary was carefully placed under the ipsilateral renal capsule of the same animal with the stromal surface adjacent to the renal cortex. In the animals undergoing ovarian ligation, the pedicle was ligated close to the ovary with $8 / 0$ prolene sutures. SCID mice were maintained on a sterile diet and water in positive-pressure isolators (Bosma et al., 1983). Human ovarian cortical biopsies $<2 \mathrm{~mm}$ thick were obtained from patients (age range 20-32 years) undergoing elective Caesarean section. This protocol was approved by the research ethics committee of the ULTH Trust. Uniformly smooth areas of cortex lacking prominent follicles or luteal tissue were selected for biopsy. The tissues were transferred to dishes containing sterile Leibovitz L-15 medium (Sigma Chemical Co., Poole) and cut into $1 \mathrm{~mm}^{3}$ cubes with the ovarian epithelium on one surface. The cubes of human ovarian tissue were randomly assigned to unilateral placement under the renal capsule of SCID mice, with the stromal surface oriented towards the kidney tissue. All of the mice made good recoveries after the operative procedures and remained healthy during the period until autopsy. At autopsy, each graft was trimmed tree of renal tissue under $\times 10$ magnification before processing as described below.

\section{Experimental design}

The experimental strategy was first to determine whether follicle survival 7 days after surgery was improved by vitamin E supplementation. By this time moribund follicles from tissue ischaemia-reperfusion would have been cleared. Second, if improvement was achieved, the strategy was to establish whether there were differences in lipid peroxidation. Finally, the investigation was to consider whether any differences in lipid peroxidation were more or less pronounced during the period of ischaemia-reperfusion.

The initial experimental design consisted of three groups: (i) 36 6-8-week-old Balb/c female mice underwent unilateral ovarian grafting (random allocation to either side) with the contralateral ovary acting as an unoperated control; (ii) the ovarian pedicle was ligated unilaterally in 18 mice of the same strain to compare the effects of complete ischaemia with the control ovary and ovarian grafts; (iii) human ovarian biopsies from nine women were grafted into female SCID mice.

Animals in each experimental group were randomly allocated to either vitamin supplement or control groups. The supplement consisted of $5 \mathrm{mg} \alpha$-tocopherol (Cambridge Labs, Newcastle) once per day s.c. starting from the day of surgery until autopsy on day 7 . On the basis of the results from the first series of murine ovarian grafts (experimental group 1), a further group of 13 animals were autografted, but with an earlier autopsy on day 3 for analysis of lipid peroxidation.

\section{Follicle counting}

The recovered grafts, ligated ovaries and control ovaries were fixed in paraformaldehyde, prepared as $5 \mu \mathrm{m}$ serial histological sections and stained with haematoxylin and eosin for follicle counting. Quantification of follicle numbers was performed by a single observer and all slides were coded for counting blindly. Every fifth section was examined and the numbers obtained were multiplied by five to allow for this procedure. The stages of follicle development were defined morphologically using the classification of Faddy et al. (1987). In the follicle counts for primordial to Graafian follicle stages, the multilaminar stages were defined on the basis of granulosa cell layers and antrum formation in the sections in which the nucleolus was visible. After decoding, the total follicle count in the graft was expressed as a percentage of that in the intact contralateral ovary of the same animal.

\section{Lipid peroxidation assays}

The grafts and ovaries were homogenized in $1 \mathrm{ml}$ sterile PBS (Gibco Labs, Paisley) on ice in $1.5 \mathrm{ml}$ Eppendorf tubes 
using Sonicaid (at $18 \mathrm{MHz}$ for $20 \mathrm{~s}$ ) and stored at $-20^{\circ} \mathrm{C}$. Murine kidney tissue was also taken for analysis. Within 4 weeks, the specimens were thawed and analysed for colorimetric (spectrophotometric) assay of total lipid peroxides and malondialdehyde (Bioxytech Kit No, LPO-586, Bioxytech, Bonneull-Sur-Marne). The detection threshold of this assay was $0.1 \mathrm{nmol} \mathrm{ml} \mathrm{m}^{-1}$ and the intra-assay variation for the SEM was $<5 \%$. Total protein was also analysed by the Lowry method (Lowry et al., 1951) so that the concentrations of total lipid peroxides and malondialdehyde could be standardized in pmol per $\mu \mathrm{g}$ of protein. An analysis of the concentrations of total lipid peroxides and malondialdehyde was carried out in the human xenografts. Limited amounts of donated tissue and high inter-graft variability, which would require a large number of observations, prohibited a study of follicle counts in the human xenografts.

\section{Statistical analysis}

The data were tested for statistical analysis using ANOVA and the Student's $t$ test (unpaired) as appropriate (using SPSS Version 6.1 for Windows).

\section{Results}

\section{Follicle counts}

On day 7 after surgery, the grafts were distinguishable from surrounding host kidney tissue by their pale coloration and morphology. From the total of 21 animals, 18 grafts were suitable for follicle counting. Three specimens were not analysed in the supplemented group because of failure to recover the graft (2 cases) or control ovary intact. Histological examination of the grafts revealed that they were vascularized with stroma and follicles that appeared to be healthy (Fig. 1a). In contrast, the ligated ovaries were largely fibrotic and follicle survival was extremely low in both supplemented and non-supplemented animals $(<1 \%$ for both groups; Fig. 1b).

The mean total follicle counts in the grafts and control ovaries of supplemented mice were $931 \pm 108$ and $1340 \pm 48$ (mean $\pm \mathrm{SEM}$ ), respectively, whereas in the nonsupplemented group they were $642 \pm 88$ and $1444 \pm 109$, respectively (Fig. 2). This represented a significant improvement in follicle survival in grafted animals receiving vitamin $\mathrm{E}$ supplementation $(P<0.05)$. As expected, there was no significant difference in the mean follicle counts between control ovaries of supplemented and nonsupplemented groups. Follicle survival in grafts was also compared as a percentage of the contralateral control ovaries. The survival rate was significantly higher in supplemented mice $(71 \pm 8 \%)$ than in non-supplemented mice $(45 \pm 6 \%)(P<0.05)$. There was no significant difference in the proportion of primordial follicles in the grafts for the supplemented animals (75 $\pm 5 \%$ ) and the non-supplemented animals $(70 \pm 3 \%)(P>0.05)$. Furthermore, these values were not significantly different compared with the control ovaries $(P>0.05)$ (Fig. 2).
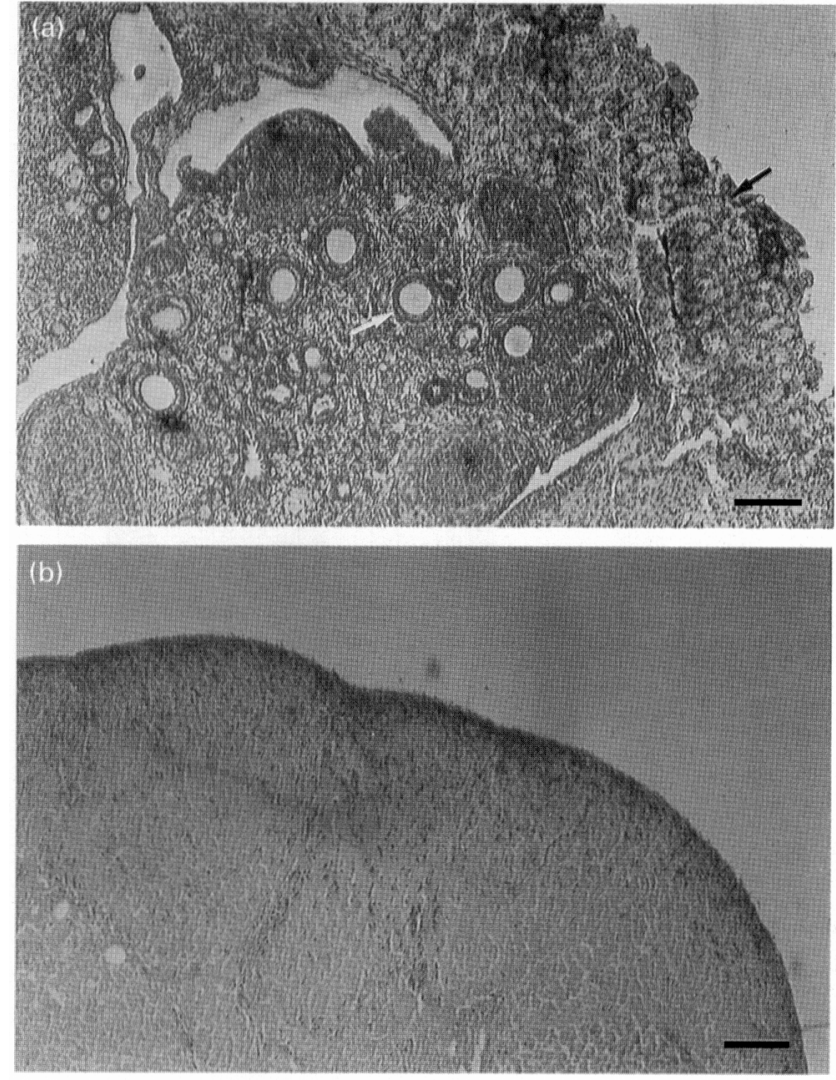

Fig. 1. (a) Histological section from a grafted murine ovary under the kidney capsule. Two-layer follicles are present within the graft (white arrow) which is positioned adjacent to the kidney tissue (black arrow). (b) Histological preparation of a ligated murine ovary illustrating a large area of ovarian cortex devoid of follicles. The sections were stained with haematoxylin and eosin. Scale bars represent $100 \mu \mathrm{m}$.

\section{Lipid peroxidation analysis}

Malondialdehyde constituted on average $69 \pm 5.7 \%$ (mean \pm SEM) of the total lipid peroxides in the ovary. The amounts of both total lipid peroxides and malondialdehyde in the murine kidney were significantly less than in the ovarian grafts $(P<0.05)$ and similar to the non-operated control ovaries. After autopsy on day 7 , there was no significant difference between the concentrations of total lipid peroxides and malondialdehyde in the isologous grafts of supplemented and non-supplemented mice, while the concentrations of both were significantly lower in the nonoperated control ovaries $(P<0.05)$ (Fig. 3a).

In contrast, a significant reduction in the amounts of both total lipid peroxides and malondialdehyde were observed in grafts of supplemented animals compared with nonsupplemented animals on day $3(P<0.05)$ (Fig. 3b). Total lipid peroxides and malondialdehyde concentrations were also significantly lower in the control ovaries compared with the grafts in non-supplemented animals $(P<0.05)$ (Fig. $3 b$ ). Total lipid peroxides and malondialdehyde concentrations were highest in the ligated ovaries at $9.8 \pm 1.1$ and $5.2 \pm 0.6$ 


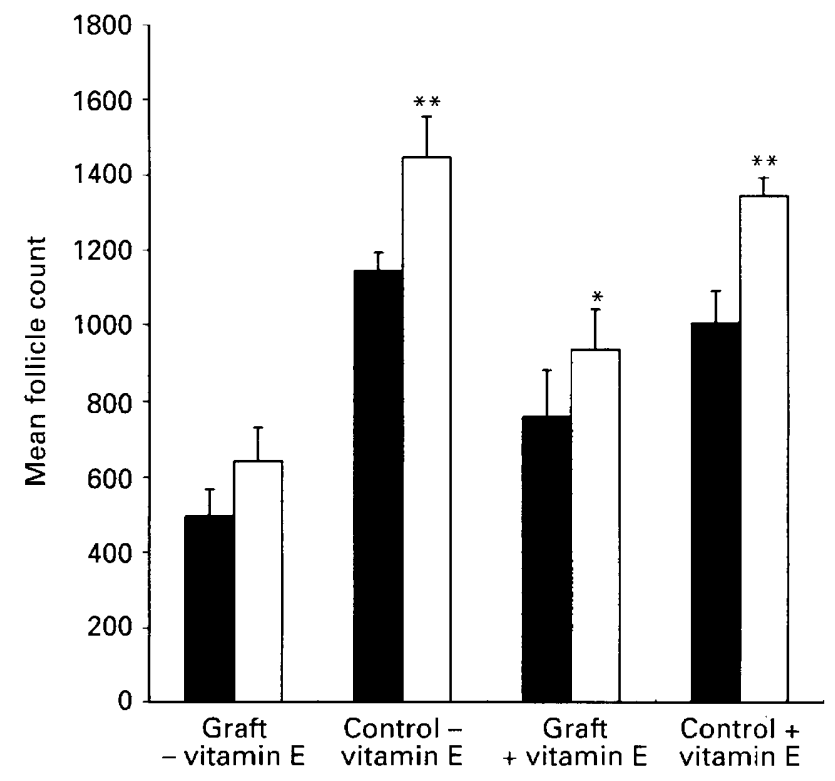

Fig. 2. The mean $( \pm$ SEM) $(\square)$ total follicle counts and the mean ( \pm SEM) (ם) primordial follicle counts in murine grafts and control ovaries on day 7 after grafting in vitamin E supplemented $(n=10)$ and non-supplemented $(n=8)$ animals. ${ }^{*}$ The mean total follicle counts are significantly higher in control ovaries compared with the grafted ovaries for both supplemented and non-supplemented animals. ${ }^{*}$ The mean total follicle count is significantly higher in the vitamin E supplemented ( $5 \mathrm{mg}$ a day s.c.) grafted animals than in the non-supplemented grafted animals $(P<0.05)$.

(mean \pm SEM) pmoles per $\mu$ g of protein, respectively, but were not significantly lowered with vitamin $E$ treatment $(P>0.05)$.

The concentrations of total lipid peroxides and malondialdehyde measured in the non-grafted human ovaries were $2.5 \pm 0.3$ and $2.3 \pm 0.2$ (mean \pm SEM) pmoles per $\mu \mathrm{g}$ of protein, respectively. In the human xenografts, vitamin E supplementation significantly reduced total lipid peroxides and malondialdehyde concentrations on day 7 $(P<0.05)$ (Fig. 4).

\section{Discussion}

This is the first study to demonstrate that lipid peroxidation occurs in ovarian grafts, and its presence indicates that ischaemia-reperfusion injury is a potential problem with this technique. Vitamin E supplementation did not affect lipid peroxidation in murine grafts on day 7 after surgery. However, this probably does not reflect the amount of lipid peroxidation in the immediate period after grafting. The ovary is naturally well-endowed with angiogenic growth factors (Koos, 1989). Studies in rats have shown that the ovarian medulla is necrotic $24 \mathrm{~h}$ after grafting, with an extensive network of vessels invading the cortex after a further $24 \mathrm{~h}$ (Dissen et al., 1994), following which reperfusion would be likely to occur. Furthermore, in mice injected i.v. with Evan's blue dye before recovery of autografts on days 1, 2 and 3 after surgery, the dye was first visible on day 3 ,
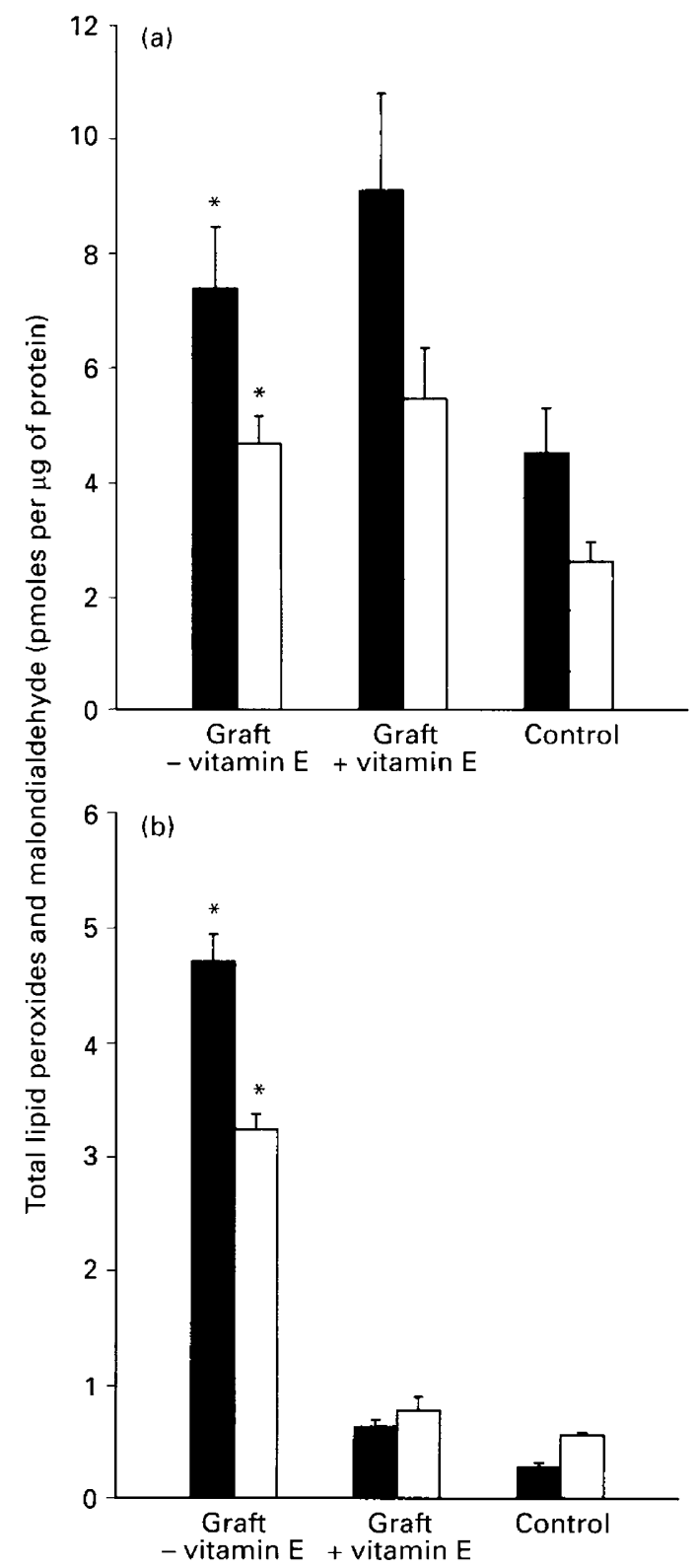

Fig. 3. (a) The mean ( \pm SEM) concentrations of ( $\square$ ) total lipid peroxides and $(\square)$ malondialdehyde in murine autografts on day 7 after grafting in vitamin E supplemented $(n=9)$ and nonsupplemented $(n=6)$ animals and in non-grafted, control ovaries $(n=7)$. ${ }^{*}$ The mean concentrations of total lipid peroxides and malondialdehyde on day 7 were significantly lower in the control ovaries compared with the grafts $(P<0.05)$. (b) The mean $( \pm$ SEM) concentrations of $(\square)$ total lipid peroxides and $(\square)$ malondialdehyde in murine autografts on day 3 after grafting in vitamin $E$ supplemented $(n=6)$ and non-supplemented $(n=7)$ animals and in non-grafted control ovaries $(n=6)$. ${ }^{*}$ The mean concentrations of total lipid peroxides and malondialdehyde on day 3 were significantly higher in the grafts of non-supplemented animals compared with the grafts in supplemented animals and control ovaries $(P<0.05)$.

indicating the presence of revascularization (D. Nugent, unpublished observations). These initial findings prompted another series of murine autografts to test whether the 


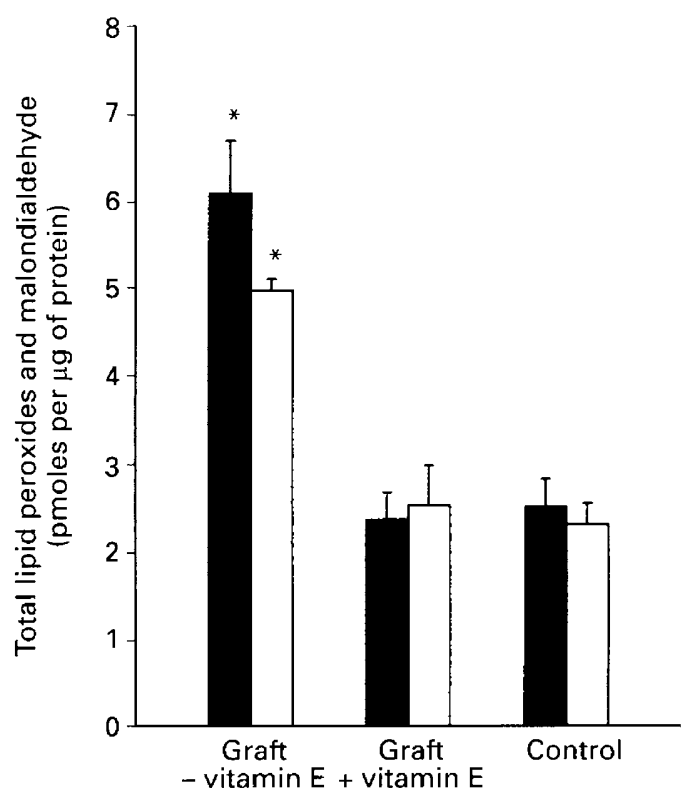

Fig. 4. The mean ( \pm SEM) concentrations of $(\square)$ total lipid peroxides and $(\square)$ malondialdehyde in human xenografts on day 7 after grafting in vitamin E supplemented $(n=5)$ and non-supplemented $(n=4)$ animals and in non-grafted control tissue $(n=5) .{ }^{*}$ The mean concentrations of total lipid peroxides and malondialdehyde were significantly higher in the xenografts of non-supplemented animals compared with the xenografts in vitamin $E$ supplemented $(5 \mathrm{mg}$ a day s.c.) animals and non-grafted human ovaries $(P<0.05)$.

antioxidant provided protection against lipid peroxidation at an earlier stage, and this was performed with autopsy on day 3. These experiments showed that the high amount of lipid peroxidation on day 3 in the grafts of non-supplemented animals were lowered significantly by administration of vitamin E. Increased amounts of total lipid peroxides and malondialdehyde after grafting, as well as their reduction with antioxidant administration, are consistent with the hypothesis that ischaemia-reperfusion injury is occurring. The dose of vitamin $E$ used in this study was supraphysiological as ischaemia-reperfusion injury is an acute abnormal physiological state and one in which shortterm high vitamin $\mathrm{E}$ supplementation may be beneficial. Although in high doses vitamin E may act as a pro-oxidant, its widespread use in diverse clinical conditions, including ischaemia-reperfusion injury, has demonstrated that these mechanisms are either insignificant in vivo or are outweighed by the protective effect of the antioxidant (Cavarocchi et al., 1986; Diplock, 1995). In human transplant recipients, the only possible limitation to the use of vitamin $E$ is potentiation of a pre-existing vitamin $K$ deficiency coagulopathy (Diplock, 1995), otherwise the safety of acute high doses has been demonstrated in both healthy human (Kappus and Diplock, 1992) and rodent toxicological studies (Krasavage and Terhaar, 1977; Abdo et al., 1986). In the present study, all of the animals were examined once a day and appeared to be healthy throughout the period between surgery and autopsy.
The highest intra-ovarian concentrations of total lipid peroxides and malondialdehyde were found in the ovaries of animals in which the ovarian pedicle was ligated as a test of acute ischaemia. This was consistent with the very high numbers of follicles lost in ovaries ligated in this way and confirmed the hypothesis that extensive lipid peroxidation is associated with significant follicular loss. On day 7 , the amount of lipid peroxidation was still high in these ovaries, with a reducing trend in supplemented animals. Vitamin supplementation did not lead to improved follicle survival after ligation, suggesting that irreversible damage had occurred. While revascularization occurs in ligated ovaries in situ, it is likely to be less rapid than in ovarian grafts in which the kidney capsule provides an environment that is highly vascularized and is known to contain high concentrations of angiogenic growth factors, such as vascular endothelial growth factor (Jakeman et al., 1992). Thus, the effects observed in the ligated ovaries may reflect either a more chronic course of ischaemia-reperfusion injury after ligation than in the grafts, or the effects of necrosis from the initial ischaemic insult.

Mice carrying the SCID mutation are congenitally immunodeficient (Bosma et al., 1983) and are a useful model for the study of xenografts, particularly those of human origin (Gosden and Aubard, 1996). In this study, human xenografts on day 7 showed significant reductions in total lipid peroxide and malondialdehyde concentrations with supplementation. Revascularization may occur more slowly within human ovarian grafts compared with murine grafts, as they are inherently more fibrous with a reduced follicle density so that the beneficial effect would be observed at a later stage. If this is the case, later revascularization may lead to a relative delay in removal of lipid peroxides and aldehydes after reperfusion.

The prospective number of menstrual cycles and the timing of the menopause is largely determined by the size of the follicle store and its subsequent rate of loss, so the conservation of germ cells in freezing and grafting procedures is a high priority (Faddy and Gosden, 1995). Thus, the increase in follicular survival from 45 to $71 \%$ after antioxidant treatment should be beneficial. While neither the long-term viability of the rescued follicles, nor the fertility of the oocytes was tested, they appeared morphologically normal without extensive atresia. Moribund cells were not seen on day 7 after grafting, suggesting that the stage of necrosis had passed and that the remaining cells, including the follicles, were probably long-term survivors. No difference was found in the proportion of primordial follicles in the grafts and control ovaries of supplemented and non-supplemented animals, suggesting that the protective effect of vitamin E occurs for all stages of follicle development. The scarcity of the tissue prevented a study of follicle counts in human xenografts although the benefits are likely to be similar, especially in view of the demonstrated reduction in lipid peroxidation.

The results of this study indicate that precautions can be taken to minimize damage resulting from lipid peroxidation during ischaemia in ovarian grafts. Other steps should also be considered, such as chilling the tissue to reduce the production of reactive metabolites before reperfusion as well as adding free radical scavengers to the medium, such as 
superoxide dismutase (Parks et al., 1982). While the use of intact ovarian transplants would sometimes be preferable to tissue slices, the practical problems of reanastomosis and whole organ cryoprotection will first have to be overcome. If this becomes possible, it is likely that antioxidants will be beneficial for whole ovaries, as they have been for kidneys (Ploeg, 1990; Schneeberger et al., 1990) and other organ transplants (Johnson et al., 1991; Mayumi et al., 1993).

The authors gratefully acknowledge support given to David Nugent by Well Being on a Research Training Fellowship and to Helen Newton by the Leukaemia Research Fund as a Research Assistant. They also thank Chris Schorah for reading the manuscript and for technical advice.

\section{References}

Abdo KM, Rao G and Montgomery CA (1986) Thirteen week toxicity study of $d$-alpha-tocopheryl acetate (vitamin E) in Fischer 344 rats Food and Chemical Toxicology 24 1043-1050

Apperley JF and Reddy N (1995) Mechanism and management of treatmentrelated gonadal failure in recipients of high dose chemoradiotherapy Blood Reviews 993-116

Bosma GC, Custer RP and Bosma MJ (1983) A severe combined immunodeficiency mutation in the mouse Nature 301 527-530

Candy CJ, Wood MJ and Whittingham DG (1997) Effect of cryoprotectants on the survival of follicles in frozen mouse ovaries Joumal of Reproduction and Fertility 110 11-19

Carroll J and Gosden RG (1993) Transplantation of frozen-thawed mouse primordial follicles Human Reproduction 8 1163-1167

Cavarocchi NC, England MD, O'Brien JF et al. (1986) Superoxide generation during cardiopulmonary bypass: is there a role for vitamin E? Journal of Surgical Research 40519-527

Diplock AT (1995) Safety of antioxidant vitamins and $\beta$-carotene American Journal of Clinical Nutrition 62 1510-1516

Dissen GA, Lara HE, Fahrenbach WH, Costa ME and Ojeda SR (1994) Immature rat ovaries become revascularized rapidly after autotransplantation and show a gonadotrophin-dependent increase in angiogenic factor gene expression Endocrinology 134 1146-1154

Esterbauer H and Cheeseman KH (1990) Determination of aldehydic lipid peroxidation products: malonaldehyde and 4-hydroxynonenal Methods in Enzymology 186 407-421

Faddy MJ and Gosden RG (1995) A mathematical model of follicle dynamics in the human ovary Human Reproduction 10770-775

Faddy MJ, Telfer E and Gosden RG (1987) The kinetics of pre-antral follicle development in ovaries of CBA/Ca mice during the first 14 weeks of life Cell Tissue Kinetics $20551-560$

Felicio LS, Nelson JF, Gosden RG and Finch CE (1983) Restoration of ovulatory cycles by young ovarian grafts in aging mice: potentiation by long term ovariectomy decreases with age Proceedings National Academy of Science $806076-6080$

Gosden RG and Aubard Y (1996) The challenges of transplantation biology. In Transplantation of Ozarian and Testicular Tissues pp 43-63. RG Landes, Austin, Texas
Gosden RG, Baird DT, Wade JC and Webb R (1994) Restoration of fertility to oophorectomised sheep by ovarian autografts stored at $-196^{\circ} \mathrm{C}$ Human Reproduction 9 597-603

Gutteridge JMC and Halliwell B (1994) Free radicals and antioxidants in ageing and disease: fact or fantasy? In Antioxidants in Nutrition, Health and Disease pp 111-123. Oxford University Press, Oxford

Harp R, Leibach J, Black J, Keldahl C and Karlow A (1994) Cryopreservation of murine ovarian tissue Cryobiology 31 336-343

Hunter MCH, Gless JP and Gazet JC (1980) Oophoropexy and ovarian function in the treatment of Hodgkin's disease Clinical Radiology 31 21-26

Jakeman LB, Winer J, Bennett GL, Altar CA and Ferrara N (1992) Binding sites for vascular endothelial growth factor are localised on endothelial cells in adult rat tissues Journal of Clinical investigation 89 244-253

Johnson WD, Kayser KL, Brenowitz JB and Saedi SF (1991) A randomized controlled trial of allopurinol in coronory bypass surgery American Heart fournal $12120-24$

Jones EC and Krohn PL (1960) Orthotopic ovarian transplantation in mice Journal of Endocrinology $20135-146$

Kappus H and Diplock AT (1992) Tolerance and safety of vitamin E: a toxicological position report Free Radical Biology and Medicine 13 55-74

Koos RD (1989) Potential relevance of angiogenic factors to ovarian physiology Seminars in Reproductive Endocrinology $729-40$

Krasavage WJ and Terhaar CJ (1977) $d$-Alpha-tocopherol-polyethyleneglycol 1000 succinate. Acute toxicity, subchronic feeding, reproduction and teratologic studies in the rat Journal of the Science of Food and Agriculture 25 273-278

Krinsky NI (1988) Membrane antioxidants Annals of the New York Academy of Science 551 17-33

Lindell SL, Gandolph D, Southard JH and Belzer FO (1991) Comparison of PBS, HTK and UW solutions for kidney preservation Transplant Proceedings $232399-2401$

Lowry OH, Rosebrough NJ, Lewis-Farr A and Randall RJ (1951) Protein measurement with the folin phenol reagent Journal of Biological Chemistry $193265-275$

Mayumi T, Schiller HJ and Bulkley GB (1993) Pharmaceutical interventions for the prevention of post-ischemic reperfusion injury. In Free radicals: from Basic Science to Medicine pp 438-457 Eds G Poli, E Albano and MU Dianzani. Birkhäuser Verlag, Berlin

Newton H, Aubard Y, Sharma V, Rutherford AJ and Gosden RG (1996) The low temperature storage and grafting of human ovarian tissue into SCID mice Huntan Reproduction 11 1487-1491

Nugent D, Meirow D, Brook PF, Aubard Y and Gosden RG (1997) Transplantation in reproductive medicine: past experience, present knowledge and future prospects Human Reproduction Update 3267-280

Parks DA, Bulkley GB, Granger DN, Hamilton SR and McCord JM (1982) Ischaemic injury in the cat small intestine: role of superoxide radicals Gastroenterology 82 9-15

Parks JE and Ruffing NA (1992) Factors affecting low temperature survival of mammalian oocytes Theriogenology 37 59-72

Ploeg RJ (1990) Kidney preservation with the UW and Euro-Collins solution a preliminary report of a clinical comparison Transplantation 49 281-284

Schneeberger H, Schleibner S, Illner WD et al. (1990) Prevention of acute renal failure after kidney transplantation by treatment with rh-SOD: interim analysis of a double-blind placebo-controlled trial Transplant Proceedings 22 2224-2225

Trounson AO and Bongso A (1996) Fertilisation and development in humans Current Topics in Developmental Biology 32 59-101 